\title{
Potential und Grenzen von «Individualisierter Medizin» (personalized medicine)
}

\author{
Schweizerische Akademie \\ der Medizinischen Wissen- \\ schaften $(S A M W)$
}

\section{* Der Entwurf dieses Positions- papiers stammt von Prof. Andreas Papassotiropoulos von der Abteilung für Molekulare Neurowissen- schaften der Universität Basel Der SAMW-Vorstand hat das Dokument an seiner Sitzung vom 3./4. September 2012 ausführlich diskutiert und überarbeitet und anschlies- send per Zirkularbeschluss in der vorliegenden Form verabschiedet.}

Korrespondenz: Schweizerische Akademie der Medizinischen Wissenschaften (SAMW) Petersplatz 13 CH-4051 Basel

mail[at]samw.ch

\section{Zusammenfassung}

Die individualisierte Medizin (IM) (oft auch als personalisierte Medizin bezeichnet) ermöglicht völlig neue Perspektiven für das Verständnis von Krankheitsentstehung und Krankheitsverlauf, aber auch neue Ansatzpunkte zur Entwicklung von Wirkstoffen. Die Fortschritte der IM waren in den letzten Jahren enorm. Gleichzeitig haben sich jedoch auch verschiedene Probleme akzentuiert, die in diesem Positionspapier benannt und für die Lösungsansätze skizziert werden. Im Moment wird die IM vor allem in folgenden Bereichen angewendet: Prädiktion; Diagnostik; Therapie (inkl. Outcome-Evaluation); Medikamentenentwicklung.

Bei der Prädiktion (Abschätzung des Krankheitsrisikos) zeigen sich einerseits das Potential wie auch die Grenzen der IM. Bei monogenen Erkrankungen kann die IM gute Voraussagen liefern. Die allermeisten Erkrankungen entstehen allerdings durch ein komplexes Zusammenspiel von mehreren Genen sowie von Umweltfaktoren. Bei oligogenen Erkrankungen (ungefähr zehn Gene beteiligt) kann eine Prädiktion zum Teil noch errechnet werden, bei polygenen Erkrankungen hingegen ist das meist nicht mehr möglich und die Aussagekraft von Gentests entsprechend beschränkt. Eine gründliche Familienanamese ist insgesamt oft aussagekräftiger als Vorhersagen aus breit angelegten genetischen Untersuchungen. In der Diagnostik spielt die IM bereits heute eine wichtige Rolle. Insbesondere in der Onkologie werden die verschiedenen Krebsarten immer häufiger gemäss ihrem «genetischen Fingerabdruck» diagnostiziert. Aber auch in anderen Bereichen ist die IM für viele Ärzte ein wertvolles, neues Diagnoseinstrument, etwa in der Kardiologie.

Auch in der Therapie hat die IM signifikante Fortschritte ermöglicht. Immer öfter werden heute Wirkstoffe zugelassen, die nur bei Patientengruppen mit entsprechenden molekularen Merkmalen wirksam sind. Vor allem in der Onkologie ist diese Entwicklung zu beobachten. Dadurch verbessert sich nicht nur die Wirksamkeit der Wirkstoffe für die betroffene Patientengruppe, sondern auch die Nebenwirkungen werden reduziert.

Bei der Medikamentenentwicklung kann es sich heute kein Pharmaunternehmen mehr leisten, die Daten der IM zu vernachlässigen. Sie werden in allen Etappen der Entwicklung berücksichtigt, auch bei der Planung von klinischen Studien. Aufgrund des rasanten Fortschritts können heute aber auch verschiedene Problembereiche eruiert werden, bei denen Handlungsbedarf besteht. Das Positionspapier benennt die Bereiche Wissensmangel, Expertenwildwuchs, unseriöse Angebote, Transparenz, Informed Consent, Datenschutz, Wirksamkeitsnachweis neuer Therapien sowie Patente und Forschungsfreiheit.

Vor dem Hintergrund dieser Probleme formuliert das vorliegende Positionspapier einige Lösungsansätze:

\section{Behebung des Wissensmangels}

Die IM steht zwar noch immer am Anfang, aber der Wissenszuwachs läuft rasant. Es ist deshalb wichtig, dass Ärztinnen und Ärzte über ausreichende Kenntnisse in den Bereichen Epidemiologie, medizinische Genetik sowie medizinische Statistik verfügen, um die Ergebnisse der IM richtig einordnen zu können. Zudem erwartet der online informierte Patient, dass ihm Ärztinnen und Ärzte mit entsprechenden Kenntnissen gegenübersitzen.

\section{Stärkung der medizinischen Genetik}

Die Genetik ist ein wichtiger Bestandteil der IM. Die Ansprüche an die Ärztinnen und Ärzte bezüglich ihrer Fähigkeit zur genetischen Analyse und Beratung nehmen zu. Eine Berücksichtigung und Stärkung der medizinischen Genetik in der medizinischen Ausbildung erscheint daher unabdingbar.

\section{Lancierung von Spezialbereichen}

Jede Erkrankung besitzt ihre eigene Genetik und Komplexität. Daher ist es notwendig, entsprechende Weiter- und Fortbildungsangebote zu fördern, damit jeder Arzt die Möglichkeit hat, das IM-relevante Wissen in seinem Gebiet zu erwerben. Patienten wiederum haben ein Recht darauf, zu erkennen, welcher Arzt über das notwendige Wissen verfügt, um IM-relevante Fragen beantworten zu können.

\section{Stärkere Gewichtung der Familienanamnese}

Dazu braucht es in der Aus- und Weiterbildung eine Sensibilisierung für die Bedeutung der Familienanamnese und darüber hinaus klinische Leitlinien zu deren Interpretation und Verwendung sowie eine intensivere Vermittlung der notwendigen Kenntnisse und Kompetenzen während des Medizinstudiums.

5. Vermeidung von Fehlentwicklungen und Schaffung von Transparenz

Frei zugängliche, IM-relevante Angebote müssen einer wissenschaftlichen Prüfung unterzogen werden, sodass jeder potentielle Nutzer weiss, worauf er sich einlässt. Dazu gehört auch die Schaffung von Transparenz bezüglich potentieller Interessenkonflikte jedwelcher Art. Nichtreglementierbare Entwicklungen bedürfen einer klaren Positionierung seitens der medizinischen Wissenschaften.

Die IM ist eine grossartige Chance, um im Sinne der Patientinnen und Patienten Prädiktion, Therapie und Nachsorge zu verbessern. Durch wissenschaftlich fundierte Kenntnisse und evidenzbasiertes, seriöses Handeln sowie durch entsprechende Schwerpunktsetzungen wird es gelingen, das Maximum aus dieser Chance herauszuholen und mögliche Fehlentwicklungen zu vermeiden. Für die Patientenbedürfnisse ist dabei entscheidend, dass die Techniken der Datenerhebung und -verarbeitung der IM die persönliche Arzt-Patienten-Beziehung nicht verdrängen, sondern in diese eingebettet werden. 


\section{Hintergrund}

Medizinische Fortschritte und technologische Errungenschaften sind seit jeher eng miteinander verflochten. Ob durch die Entwicklung neuer histologischer Färbemethoden, die Nutzung von elektromagnetischen Wellen oder die Herstellung neuartiger Metalllegierungen - um nur einige Beispiele zu nennen -, hat die Technologie der Medizin immer wieder neue Perspektiven eröffnet.

Seit über zehn Jahren erleben wir im biomedizinischen Bereich erneut eine Revolution, die auf zwei parallelen Entwicklungen beruht. Einerseits stehen immer schnellere, hochauflösende und übergreifende molekulare Analysen zur Verfügung, die auch zunehmend kostengünstiger werden (z.B. genomweite Polymorphismusanalysen, genomweite DNA- und RNA-Sequenzierung, Epigenomik, Proteomik, Metabolomik). Andererseits macht die Entwicklung integrativer analytischer Methoden im Bereich der Bioinformatik die enorme Datenflut einer sinnvollen Interpretation besser zugänglich.

Dadurch eröffnen sich völlig neue Perspektiven für das Verständnis physiologischer und pathophysiologischer Prozesse, der Krankheitsentstehung, des Krankheitsverlaufs und der therapeutischen Wirkung. Diese neuen Informationen und Erkenntnisse werden zudem schnell und teilweise ungefiltert über das Internet für jede Person überall auf der Welt abrufbar. Die Kombination aus komplexen technologischen Entwicklungen, deren Anwendung in der medizinischen Wissenschaft sowie der globalen Verfügbarkeit sämtlicher Informationen wird die Art und Weise, wie wir über Gesundheit und Krankheit denken, und damit die gesamte Medizin verändern. Unweigerlich wird es dadurch zu Umwälzungen und zum Aufkommen vieler Fragen wissenschaftlicher, ethischer und gesundheitspolitischer Natur kommen. Es ist also an der Zeit, sich auch in der Schweiz mit diesen Entwicklungen und den sich daraus ergebenden Fragen aktiv auseinanderzusetzen. Der medizinischen Wissenschaft kommt dabei die Aufgabe zu, eine evidenzbasierte sowie ethisch fundierte Meinungsbildung zu fördern.

\section{Definition}

Die eingangs erwähnten Fortschritte in den sogenannten «omics»-Technologien und die damit verbundene Möglichkeit, von einem Individuum eine Vielzahl medizinischer Informationen - inklusive kompletter Genomsequenzen - zu erhalten und teilweise zu interpretieren, hat zur vermehrten Verwendung des Begriffs der «personalisierten Medizin» geführt. Ebenfalls verwendet werden die Begriffe «individualisierte», «massgeschneiderte», «stratifizierte» oder «Präzisions»-Medizin. Da der Begriff «personalisierte Medizin» insofern etwas irreführend ist, als das damit beschriebene medizinische Vorgehen nicht auf die personenspezifischen Eigenschaften eines Menschen abzielt, sondern vielmehr auf die individuellen biologischen Strukturen und Prozesse, verwenden wir in diesem Positionspapier den Begriff der «individualisierten Medizin» (IM).

Das vorliegende Positionspapier versteht unter IM ein medizinisches Vorgehen, bei dem die Daten eines Individuums auf molekularer Ebene erhoben und diese mit Mitteln der Informationstechnologie im Hinblick auf eine individualisierte Prognosestellung, Beratung und/oder Therapie ebendieses Individuums ausgewertet werden.

Je nach Art der erhobenen Daten kann diese Definition enger gefasst werden (z.B. durch Beschränkung auf genomische Daten, die sogenannte «genomische Medizin») oder durch den Miteinbezug anderer Daten erweitert werden (z. B. anamnestische bzw. krankheitsbezogene Daten, die sogenannte «datenbasierte Medizin»).

Das beschriebene Vorgehen verdient den Namen «Medizin» jedoch nur dann, wenn der Prozess bereits vor Beginn der Datenerhebung und über die Erstellung einer Prognose oder Empfehlung hinaus betrachtet wird. Damit IM der Persönlichkeit des Patienten oder des Nutzers gerecht wird, braucht es mehr als rohe Daten, nötig ist die persönliche Beziehung zu einer Fachperson bzw. einem Arzt. Beim Einsenden einer biologischen Probe (allenfalls begleitet von einem ausgefüllten Fragebogen) und dem darauf folgenden Empfang einer gedruckten Empfehlung handelt es sich somit (noch) nicht um IM.

Der exponentielle Zuwachs des Gebrauchs der oben genannten Begriffe suggeriert, dass es sich möglicherweise um ein neuartiges Konzept in der Medizin handelt. Obwohl die Methodik, der sich die IM bedient, tatsächlich neuartig ist, ist der zugrundeliegende Gedanke so alt wie die Medizin selbst. Eine «individualisierte Medizin» (IM) bezweckt die Optimierung der medizinischen Versorgung für jedes einzelne Individuum von der Prophylaxe bis hin zur Therapie [1], indem sowohl individuelle biologische Eigenschaften, Umweltfaktoren und kontextuelle Einflüsse über die Lebensspanne eines Individuums hinweg erfasst und analysiert werden. Tatsächlich beschreibt dieser Zweck der IM das medizinische Denken mindestens seit Hippokrates. Die Fokussierung auf das Individuum mit seiner Einzigartigkeit ist Charakteristikum jedes korrekten ärztlichen Handelns. Ein Blick in die Datenbanken zeitgenössischer medizinischer Literatur zeigt, dass die Verwendung des Begriffs der «personalisierten Medizin» seit dem Anfang dieses Jahrhunderts einen exponentiellen Anstieg aufweist. Allerdings gibt es bereits vereinzelte Berichte in den 1970er und 1990er Jahren, die sich dem Thema widmen. In diesen Veröffentlichungen wird sogar der Niedergang der IM, d.h. der Fokussierung auf das Individuum und der individuellen Beratung, befürchtet, und zwar als Folge der zunehmenden Technisierung und «Algorithmisierung» der Medizin [2, 3]. In diesen Bedenken kommt die Einsicht zum Tragen, dass die menschliche Person mehr ist als die Summe der Daten, die über sie erhoben werden können. Sie kann ihrem Wesen nach nur in der Begegnung mit einer anderen Person erfasst werden. Es ist deshalb 
entscheidend, dass die Techniken der Datenerhebung und -verarbeitung der IM die persönliche Arzt-Patienten-Beziehung nicht verdrängen, sondern in diese eingebettet werden.

\section{Anwendungsbereiche}

\section{der «individualisierten Medizin»}

Wie oben ausgeführt, ist das Konzept der IM weder neu noch aussergewöhnlich, sondern stellt eine Selbstverständlichkeit im medizinischen Denken dar. Dennoch ist die Medizin an einem Punkt angelangt, an dem die technologischen und analytischen Entwicklungen das Repertoire der präventiven, diagnostischen und therapeutischen Möglichkeiten enorm vergrössern und teilweise diagnostische und therapeutische Prozesse auf den Kopf stellen. Deswegen ist es zunächst hilfreich, die Anwendungsbereiche der modernen IM zu umreissen. Da der Bereich der Genomik momentan die schnellsten und umfassendsten sowie für die medizinische Praxis konkretesten Fortschritte aufweist, wird auf ihn besonders häufig Bezug genommen. Bereits jetzt weisen aber auch die Bereiche der Genexpression, Epigenomik, Proteomik und Metabolomik ähnliche Fortschritte auf. Diese werden aber voraussichtlich den Anwendungs- bzw. Gültigkeitsbereich der IM nicht grundlegend modifizieren.

Es lassen sich momentan vier Anwendungsbereiche der IM umschreiben: 1. Prädiktion, 2. Diagnostik, 3. Therapie, inkl. Outcome-Evaluation, 4. Entwicklung neuer Medikamente.

\subsection{Prädiktion}

Prädiktion bezeichnet die präsymptomatische Risikoabklärung und Diagnostik (inkl. pränataler Diagnostik), mit dem Ziel einer frühen Prognose, Diagnose und eventuell Therapie bzw. präventiver Massnahmen [4]. Vereinzelt ist die genombasierte Prädiktion bereits heute Teil unseres medizinischen Versorgungssystems, z. B. bei Chorea Huntington. Angesichts der Auswirkungen nicht nur auf eine einzelne Person, sondern auf die gesamte Familie, die eine genombasierte Prädiktion eines genetisch dominanten Merkmals erhält, ist der vom Gesetzgeber festgeschriebene Beratungsaufwand zwar erheblich, jedoch richtig und notwendig. Es ist nicht das Ziel dieses Positionspapiers, auf die ethische Dimension einer genombasierten Prädiktion einzugehen. Gleichwohl ist es wichtig und gerade im Licht eines Überangebots an sogenannten prädiktiven Tests sogar unerlässlich, festzuhalten, wann überhaupt eine genombasierte Prädiktion wissenschaftlich begründbar und machbar ist.

Generell ist festzuhalten, dass es sich bei jeder Art der Prädiktion (auch der genombasierten) um eine probabilistische Aussage handelt. Die korrekte Vermittlung von Wahrscheinlichkeiten ist jedoch ein komplexes Unterfangen [5, 6].

Die Güte einer prädiktiven Aussage leitet sich von der Effektstärke des Prädiktors ab. Die IM kann daher bei monogenen Erkrankungen, bei denen die Effektstärke einer genetischen Variation per definitionem hoch ist, einen signifikanten Beitrag leisten. Bei oligogenen Erkrankungen, die durch eine kleine Zahl genetischer Variationen (selten mehr als 10) bedingt sind (z.B. einige Krebsarten), ist eine akkurate Prädiktion weitaus schwieriger. Dies zum einen, weil bei oligogenen Erkrankungen Umweltfaktoren eine grosse Rolle spielen, zum anderen können die krankheitsassoziierten Varianten miteinander interagieren, was die Berechnung verkompliziert und eine akkurate prädiktive Aussage deutlich erschwert oder verunmöglicht. Dennoch lassen sich durch genetisch-epidemiologische Studien, verbunden mit genom-weiten Analysemethoden, die Effektstärken und die Gen-Gen-Interaktionseffekte modellieren und oft im Sinne einer Prädiktion errechnen, wobei die Güte der prädiktiven Aussagen naturgemäss im Vergleich zu den monogenen Erkrankungen weitaus geringer ist (z.B. [7])

Diese Problematik ist bei polygenen Erkrankungen (z. B. koronare Herzkrankheit, arterielle Hypertonie, Demenz, psychiatrische Erkrankungen) akzentuiert. Die Vielzahl der risiko-assoziierten genetischen und nichtgenetischen (Umwelt-)Faktoren bzw. ihr äusserst komplexes Interaktionsmuster verunmöglichen in den meisten Fällen eine seriöse Prädiktion auf individueller Ebene. Es darf nicht vergessen werden, dass auch ein bedeutender genetischer Risikofaktor, der anhand von gruppenstatistischen Analysen identifiziert wurde (z.B. das Apolipoprotein-E4-Allel bei der Alzheimer-Demenz) unter Umständen wenig hilfreich ist, wenn es darum geht, eine individuelle Vorhersage zu treffen [8, 9]. Zusätzlich ist zu beachten, dass jede Erkrankung ihre eigene Genetik und Komplexität besitzt. Die Genetik der arteriellen Hypertonie ist anders als die Genetik des Pankreaskarzinoms, und diese ist wiederum anders als die der bipolaren affektiven Störung, selbst wenn diese Erkrankungen alle unter dem Begriff der polygenen Erkrankungen subsumiert werden können.

In den letzten Jahren haben hochauflösende, genomweite Assoziationsstudien (genome-wide association studies, GWAS), die Millionen von genetischen Polymorphismen in sehr grossen Fall-KontrollKollektiven untersuchen (teilweise über 100000 Teilnehmer), eine Vielzahl an replizierten genetischen Risikofaktoren und Suszeptibilitätsgenen für verschiedene polygene Erkrankungen identifiziert. Diese Studien bedeuten einen grossen Fortschritt für das Verständnis der Pathophysiologie der entsprechenden Erkrankungen und haben bereits zu neuen therapeutischen Entwicklungen geführt [10].

Wenn es allerdings um die Berechnung des individuellen Erkrankungsrisikos anhand dieser identifizierten Varianten geht - ein Versprechen, das gewisse Firmen werbewirksam propagieren -, dann sehen die Resultate ernüchternd aus: Trotz des massiven Einsatzes an breit angesetzten, genetischen Untersuchungen erfüllen die identifizierten Varianten die Krite- 
rien, die für prädiktive Tests gelten, bei weitem nicht. Dieses Resultat erstaunt angesichts der Komplexität polygener Erkrankungen nicht. Interessant allerdings ist die wiederholte Beobachtung, dass jeder noch so signifikante «prädiktive» Effekt der genetischen Risikovarianten statistisch gesprochen zunichte gemacht wird, wenn im Modell die Familienanamnese miteinbezogen wird (z.B. [11]).

In anderen Worten: Geht es um die Abschätzung des individuellen Risikos einer polygenen Erkrankung, so ist eine gründliche Familienanamnese einer genetischen Testung risikoassoziierter Varianten weitaus überlegen. Die gründliche Familienanamnese berücksichtigt immer die Gesamtheit aller genetischen und nicht-genetischen (jedoch geteilten) Risikofaktoren innerhalb der Familie sowie das komplexe Interaktionsmuster dieser Faktoren, und ist somit viel aussagekräftiger als jede noch so breit angelegte, aber letztlich isolierte genetische Untersuchung. Mit der gründlichen Familienanamnese hat jede praktizierende Ärztin und jeder praktizierende Arzt ein sehr wirksames Instrument in der Hand, um ganz im Sinn der IM eine fundierte und seriöse Risikoabschätzung vorzunehmen. Leider wird die Anamnese (sowohl die familiäre als auch die persönliche) im praktischen Medizineralltag oft vernachlässigt und ist nicht optimal in die Gesundheitsversorgung integriert [12].

\subsection{Diagnostik}

Der Beitrag der IM in der Diagnostik und der Prognose von Erkrankungen ist bereits heute signifikant, und das Entwicklungspotential ist sehr hoch. Als Vorreiter gilt die Onkologie, die sich moderner IM-basierter Methoden bedient, um zu einer molekularen Diagnostik und zu einer molekularen Charakterisierung von Malignomen zu gelangen. Der Vorteil der Onkologie besteht darin, dass genomische, epigenomische, und proteomische Untersuchungen direkt am Tumorgewebe vorgenommen werden können und eine detaillierte Subtypisierung der jeweiligen Erkrankungen ermöglichen. Ob im Bereich des Mammakarzinoms, des Kolonkarzinoms, der Lymphome, Leukämien usw., IM hat zu einem grossen und auch für die Patientinnen und Patienten relevanten Wissenszuwachs geführt. Mehrere komplette Genome von Malignomen wurden bereits sequenziert, etwa bei akuter myeloischer Leukämie und nichtkleinzelligen bzw. kleinzelligen Bronchuskarzinomen [13-15]. Es gibt grosse internationale Konsortien, die sich der kompletten Charakterisierung von Tumorgewebe - von der DNA zum Protein - widmen, so z. B. beim Glioblastom und beim Mammakarzinom.

Zusätzlich zur Onkologie nutzen aber auch andere Fachbereiche die IM, um das diagnostische und prognostische Repertoire zu vergrössern, wie z.B. die Kardiologie. Anhand metabolomischer und proteomischer Ansätze wird untersucht, ob zwischen den verschiedenen Stadien eines akuten Myokardinfark- tes und einer instabilen Angina pectoris unterschieden werden kann $[16,17]$. Zusätzlich werden verschiedene, auf Genexpression im Blut basierende Tests validiert, um den Schweregrad einer koronaren Herzerkrankung zu quantifizieren [18, 19]. Zusammenfassend kann man festhalten, dass die IM sehr wohl wichtig ist, um die Diagnostik (inkl. Prognose) einer Vielzahl von Erkrankungen im Sinne der Patientinnen und Patienten zu verbessern.

\subsection{Therapie (inkl. Outcome-Evaluation)}

Die IM ist in diesem Bereich weit fortgeschritten. Auch hier hat die Onkologie eine Vorreiterrolle, vor allem in der Berücksichtigung pharmakogenetischer Parameter. Die Entwicklungen in diesem Bereich der Therapie und der Outcome-Evaluation gehen selbstverständlich Hand in Hand mit den Entwicklungen im Bereich der Diagnostik, d.h. der besseren Subtypisierung aufgrund molekularer Merkmale des Tumors. So werden zur Behandlung verschiedener Krebsarten zunehmend Substanzen zugelassen, die nur bei bestimmten molekularen Untergruppen wirksam sind [4]. Auch hier gibt es gross angelegte Programme (z. B. in Frankreich, den USA, Norwegen und Grossbritannien) mit dem Ziel, anhand einer kompletten molekularen Charakterisierung von Tumorbiopsien zu individuell wirksamen Therapien zu gelangen. Diese Entwicklungen sind nicht nur für die Wirksamkeit von Relevanz, sondern auch für die Vermeidung von unnötigen und potentiell schädlichen Behandlungen, wie auch für die Evaluation des therapeutischen Outcomes. Und auch hier wird nicht nur die Onkologie profitieren, sondern prinzipiell alle klinischen Gebiete, wie z. B. das Spektrum der kardiovaskulären, metabolischen, neuropsychiatrischen oder dermatologischen Erkrankungen. Die IM wird wenn richtig eingesetzt - zu einer signifikanten Verbesserung der therapeutischen Optionen in der gesamten Medizin beitragen.

\subsection{Entwicklung neuer Medikamente}

Die Anwendung IM-relevanter Methoden ist auch für die Entwicklung neuer Medikamente von grösster Wichtigkeit. An jedem Punkt der Medikamentenentwicklung (target identification, target validation, lead development, preclinical phases, clinical phases, market) ist die Rolle von Genomik, Epigenomik und Proteomik zentral [1]. Zudem wird evident, dass schon vor Einführung eines neuen Medikamentes die selektive Wirksamkeit anhand IM-basierter Methoden zu prüfen ist. Zwar werden so die dafür notwendigen klinischen Studien (insbesondere die Phase-III-Studien) noch aufwendiger. Auf der anderen Seite führt die IM-basierte Stratifizierung von Probanden zu höheren Effektstärken, sodass die zu rekrutierende Teilnehmerzahl für die Studie gesenkt werden kann. Es ist offensichtlich, dass die IM den Bereich der Medikamentenentwicklung signifikant und im Sinne der Patientinnen und Patienten positiv beeinflussen wird. Im Prinzip berücksichtigt heute 
jedes pharmazeutische Unternehmen die IM bei der Entwicklung neuer Medikamente für jeden Bereich der klinischen Medizin.

\section{Problembereiche}

Wie bei jedem technologischen Fortschritt tauchen auch bei der IM viele Fragen auf, die möglichst frühzeitig diskutiert werden müssen, um Fehlentwicklungen vorzubeugen. Nachfolgend werden acht Problembereiche aufgeführt, die besonderer Beachtung bedürfen.

\subsection{Wissensmangel}

Die Bedeutung und auch die Aussagen der IM lassen sich ohne statistisches und epidemiologisches Wissen nicht verstehen bzw. nicht korrekt interpretieren. Bei der IM dreht sich alles um probabilistische Aussagen, die immer im jeweiligen Kontext zu verstehen sind. Eine 80-prozentige Wahrscheinlichkeit für das Ansprechen auf ein bestimmtes Medikament und eine 80-prozentige Auftretenswahrscheinlichkeit einer unheilbaren Erkrankung haben sehr unterschiedliche Bedeutung für das betroffene Individuum. Die statistische Signifikanz eines Suszeptibilitätsgens besagt noch nichts über seine prädiktive Wertigkeit. Ein Risikoallel mit einer «odds ratio» von beispielsweise 3 kann dennoch für die Prädiktion des entsprechenden Merkmals unbedeutend sein. Erstaunlicherweise deckt gerade die Debatte rund um die IM bei verschiedenen Beteiligten eklatante Wissenslücken bezüglich einfacher statistisch-epidemiologischer Prinzipen auf. Es gilt, diese Wissenslücken möglichst schnell und systematisch zu schliessen. Zudem gilt es, der Vernachlässigung der (Familien-) Anamnese - wie oben erläutert - mit konkreten Massnahmen entgegenzuwirken.

\subsection{Expertenwildwuchs}

Die IM umfasst ein breites Wissensgebiet mit Aspekten der Medizin, Biologie, Biotechnologie, Informatik und Ethik. Dies hat zur Folge, dass der akademische Diskurs um die IM ausgesprochen pluridisziplinär gestaltet wird, was gut und erwünscht ist. Es darf allerdings nicht vergessen werden, was Sinn und Zweck der IM ist: Die IM bezweckt die Optimierung der medizinischen Versorgung für jedes einzelne Individuum von der Prophylaxe bis hin zur Therapie. Da die Stimme der Experten in diesem für die Medizin sehr wichtigen Bereich Auswirkungen haben kann, ist es an der Zeit zu realisieren, dass selbsternanntes Expertentum potentiell schädlich ist und an IM-Experten gewisse Mindestanforderungen zu stellen sind.

\subsection{Unseriöse Angebote}

«Sie sind Trägerin einer Genvariante, die zu mehr Intelligenz führt, falls Sie gestillt wurden.» Das Angebot solcher «prädiktiver» Tests ist geeignet, die IM in ihrer Gesamtheit zu diskreditieren. Tatsächlich pro- pagieren bekannte international tätige Anbieter solche sogenannten «direct-to-consumer»-(DTC) Gentests, genauso wie genetische Tests zur Vorhersage eines guten oder schlechten Gedächtnisvermögens, der Schizophrenie oder des Hangs zu suizidalem Verhalten. Diese unwissenschaftlichen «Angebote» offenbaren ein Problem: Im Bereich der IM hat sich ein Markt etabliert, der unkontrollierbar und unregulierbar ist. In der Schweiz sind solche DTC-Tests ohne ärztliche Anweisung grundsätzlich verboten. Verbote nützen hier jedoch wenig, weil sie in einer globalisierten Welt denkbar einfach umgangen werden können. Umso wichtiger ist es daher, diese Angebote einer wissenschaftlichen und für alle sichtbaren und abrufbaren Prüfung zu unterziehen, sodass jeder potentielle Nutzer dieser Angebote weiss, worauf er sich einlässt. Eine Reihe nationaler und internationaler Fachgruppen hat sich bereits eindeutig positioniert und vor solchen Angeboten gewarnt. Beispielhaft dafür steht das entsprechende Merkblatt der Expertenkommission für genetische Untersuchungen beim Menschen (GUMEK) zu Gentests aus dem Internet (www.bag.admin.ch/gumek).

Zusätzlich zur Problematik der Unwissenschaftlichkeit mancher Angebote offenbaren die Entwicklungen im Bereich der DTC-Gentests ein anderes Problem: den Mangel an genetischer Beratung (siehe dazu auch 4.5.). Manche DTC-Angebote sind durchaus valide, wie z.B. die Bestimmung von Mutationen bei dominant oder rezessiv vererbten Merkmalen (z.B. Chorea Huntington). Die potentiellen Nutzer erfahren ihre Ergebnisse allerdings ungefiltert und unmittelbar ohne notwendige Zusatzinformationen und ohne entsprechende Beratung. Es ist fahrlässig, diesen Umstand mit dem - selbstverständlich nicht in Frage zu stellenden - Recht auf Freiheit der Einholung von Information zu verharmlosen. Vielmehr handelt es sich um eine ethisch nicht akzeptable Unterlassung, da es sich hierbei nicht um irgendeine Information handelt, sondern um komplexe medizinische Sachverhalte, die einer umsichtigen Interpretation bedürfen.

\subsection{Transparenz}

Die IM ist nicht nur wissenschaftlich von höchstem Interesse, sondern auch wirtschaftlich. Da es sich um eine zukunftsweisende Richtung der Medizin handelt, ist der potentielle wirtschaftliche Nutzen signifikant. Gerade deswegen und weil es um die Gesundheit jedes Einzelnen geht, muss grösstmögliche Transparenz gelten. Ob Experten, einflussreiche Diskutanten, Anbieter von IM-Leistungen, Initianten von öffentlichen Diskussionsplattformen und internetbasierten Foren - sie alle müssen ihr persönliches Interesse begründen und etwaige wirtschaftliche Verflechtungen deklarieren. Dies ist für einen so sensiblen Bereich zwar eine Selbstverständlichkeit, deren Umsetzung muss aber sehr ernsthaft angegangen werden. 


\subsection{Informed Consent}

Wenn in einem Schritt und ohne klare medizinische Fragestellung eine sehr grosse Zahl von möglicherweise gesundheitsrelevanten Daten erhoben werden soll, muss die Forderung nach einer umfassenden und verständlichen Aufklärung über Nutzen und Risiken dieser Massnahme in neuem Licht gesehen werden. Selbstverständlich besteht bei genetischen Untersuchungen bereits heute die Pflicht, über die Möglichkeit unerwarteter Resultate von unklarer Bedeutung aufzuklären; bisher waren diese aber im Vergleich zum eigentlichen Ziel der Untersuchung stets von untergeordneter Bedeutung. Die neuen Testmöglichkeiten bringen eine solche Fülle potentiell relevanter Informationen mit sich, dass eine umfassende Aufklärung schon rein quantitativ problematisch ist. Die Tatsache, dass die Bedeutung sehr vieler Informationen noch unklar ist und sich in naher Zukunft ändern kann, kompliziert die Beratung zusätzlich. Die Frage, welche Anforderungen im Rahmen der IM an eine gültige informierte Einwilligung gestellt werden müssen, bedarf einer sorgfältigen ethischen und juristischen Klärung.

Besonders schwierig $\mathrm{zu}$ beantworten ist diese Frage, wenn nicht urteilsfähige Personen untersucht werden sollen, insbesondere Kinder und Neugeborene, sowie bei pränatalen Untersuchungen oder bei Vaterschaftstests.

\subsection{Datenschutz}

Die Informationen und Daten, die im Rahmen der IM erhoben werden, sind definitionsgemäss sehr persönlicher Natur und sensibel. Sie sind deshalb besonders schützenswert. Das involvierte, nicht nur medizinische Personal ist sich dieser Verantwortung noch nicht vollständig bewusst. Internetbenutzer sind auf die Gefahren der Verwendung solcher sensibler Daten (eigener und fremder!) ausdrücklich hinzuweisen.

\subsection{Wirksamkeitsnachweis neuer Therapien}

Bei der Wirksamkeitsprüfung neuer Medikamente erlaubt die IM eine Stratifizierung der Probanden in unterschiedliche Subgruppen. Wenn eine Substanz in der Gesamtpopulation keine, in einer Subpopulation aber eine gute Wirksamkeit zu zeigen scheint, entsteht das Problem, inwiefern der Wirksamkeitsnachweis für diese möglicherweise kleine Subpopulation als genügend angesehen werden kann. Die Möglichkeit, für solche Subpopulationen einen «orphan disease»-Status zu beanspruchen, müsste an den sorgfältigen Nachweis klinischer Wirkung und eines angemessenen Nebenwirkungsprofils geknüpft sein.

\subsection{Patente und therapeutische bzw. Forschungs- freiheit}

Es ist vorstellbar, dass der Hersteller einer Substanz ein Paket, bestehend aus einer bestimmten diagnostischen Datenerhebung, einem verknüpfenden Algorithmus und einer darauf basierenden individuali- sierten Rezeptur patentieren lässt. Dies könnte einerseits die therapeutische Freiheit des behandelnden Arztes beeinträchtigen, z.B. die Behandlung anderer, vom Algorithmus nicht erfassten Faktoren einzubinden; andererseits und vor allem könnte ein solches Patent die Forschung zur Verbesserung der individualisierten Therapie dieser Erkrankung behindern.

\section{Lösungsansätze}

Die im Folgenden vorgeschlagenen Massnahmen ergeben sich aus den oben beschriebenen Problembereichen und verstehen sich nicht als abschliessend. Die IM steht immer noch am Anfang, und es ist davon auszugehen, dass mit der Zeit zusätzliche und anders gelagerte Diskussionspunkte auftreten werden, die dann einer entsprechenden Evaluation bedürfen. Dennoch ergibt sich bereits heute die Notwendigkeit zu handeln.

\subsection{Behebung des Wissensmangels}

Die Kenntnisse der Ärzteschaft im Bereich Epidemiologie, medizinische Genetik und medizinische Statistik sind zu verbessern. Viele Fragen rund um die IM haben mit statistischen und epidemiologischen Zusammenhängen zu tun. Zudem muss uns bewusst sein, dass im Internetzeitalter die Mehrheit der Patientinnen und Patienten das Internet konsultiert und vom behandelnden Arzt Erklärungen, Interpretationen und Antworten erwartet. Ohne entsprechende Kenntnisse wird man unnötige Verunsicherung und Ängste provozieren.

\subsection{Stärkung der medizinischen Genetik}

Die Untersuchung und Interpretation von Genomsequenzen ist ein wichtiger Bestandteil der IM. Daher kommen auf alle Ärztinnen und Ärzte, die Patienten behandeln, zunehmende Ansprüche an ihre Fähigkeit zur genetischen Beratung zu. Eine entsprechende Berücksichtigung und Stärkung der medizinischen Genetik in der medizinischen Ausbildung erscheint daher unabdingbar.

\subsection{Lancierung von Spezialbereichen}

Die IM bringt eine weitere Spezialisierung bzw. Subspezialisierung mit sich. Je nach medizinischem Fachbereich unterscheidet sich die IM deutlich. Wie oben erwähnt, besitzt jede Erkrankung ihre eigene Genetik und Komplexität. Es ist heute bereits Realität, dass allgemeines genetisches Wissen nicht mehr ausreicht, um der Untersuchung der genetischen Komplexität jeder einzelnen polygenen Erkrankung gerecht zu werden, bzw. mit der Geschwindigkeit des Erkenntnisgewinns Schritt zu halten. Auf der anderen Seite wird in Kürze jeder Hausarzt, Kardiologe, Chirurge, Psychiater, Dermatologe usw. mit Patientenfragen konfrontiert werden, die sich im entsprechenden Bereich rund um die IM drehen. Daher ist es notwendig, entsprechende Weiter- und Fortbildungsangebote zu fördern, damit jeder Arzt die Möglichkeit 
hat, das IM-relevante Wissen in seinem Gebiet zu erwerben, um die Patienten entsprechend beraten $\mathrm{zu}$ können, ohne dass er aber eine Weiterbildung im gesamten Bereich der medizinischen Genetik erwerben muss. Die Patienten wiederum haben ein Recht darauf zu erkennen, welcher Arzt tatsächlich über das notwendige Wissen verfügt, um IM-relevante Fragen beantworten zu können. Letztlich wird IM-relevantes Wissen und seine fortwährende Aktualisierung zum festen Bestandteil jedes ärztlichen Spezialgebietes werden.

\subsection{Stärkere Gewichtung der Familienanamnese}

Dazu braucht es in der Aus- und Weiterbildung eine Sensibilisierung für die Bedeutung der Familienanamnese und darüber hinaus klinische Leitlinien $\mathrm{zu}$ deren Interpretation und Verwendung sowie eine intensivere Vermittlung der notwendigen Kenntnisse und Kompetenzen während des Medizinstudiums.

\subsection{Vermeidung von Fehlentwicklungen und Schaffung von Transparenz}

Wie oben ausführlicher dargelegt, gibt es nichtreglementierbare Entwicklungen, die dennoch einer klaren Positionierung seitens der medizinischen Wissenschaften bedürfen. Dies soll deutlich und sichtbar erfolgen. IM-relevante Angebote, die frei zugänglich sind, müssen einer wissenschaftlichen und für alle zugänglichen Prüfung unterzogen werden, sodass jeder potentielle Nutzer dieser Angebote weiss, worauf er sich einlässt. Hier sind die medizinischen Wissenschaften besonders gefordert. Dazu gehört auch die Schaffung von Transparenz bezüglich potentieller Interessenkonflikte jedwelcher Art.

\section{Ausblick}

Die IM verändert laufend die Art und Weise, wie wir über Medizin denken. Sie ist eine grossartige Chance, um im Sinne der Patientinnen und Patienten Prädiktion, Therapie und Nachsorge zu verbessern. Nur durch wissenschaftlich fundierte Kenntnisse und evidenzbasiertes, seriöses Handeln und durch entsprechende Schwerpunktsetzungen bereits in der Aus- und Weiterbildung aber auch in der Fortbildung wird es gelingen, das Maximum aus dieser Chance nutzbringend herauszukristallisieren und den Menschen zugutekommen zu lassen sowie drohende Fehlentwicklungen zu vermeiden.

Obwohl die IM primär auf der individualmedizinischen Ebene ansetzt, muss auch ihre Bedeutung für die Medizin als Ganzes bzw. für den Public-HealthBereich betrachtet werden. Einerseits können sich aus der Sammlung grosser Datenmengen interessante Forschungsansätze ergeben, andererseits müssen die Auswirkungen einer neuen Vielzahl individualisierter Empfehlungen auf das Gesundheitsverhalten der Bevölkerung sorgfältig monitorisiert werden. Ebenso bedürfen die Auswirkungen eines erhöhten Beratungsbedarfs sowie die mit der IM verbundenen ethischen Fragen einer vertieften Analyse.

\section{Literatur}

1 Chan IS, Ginsburg GS. Personalized medicine: progress and promise. Annu Rev Genomics Hum Genet. 2011;12:217:44.

2 Gibson WM. Can personalized medicine survive? Can Fam Physician. 1971;17:29-88.

3 Arnold RM, Forrow L. Rewarding medicine: good doctors and good behavior. Ann Intern Med. 1990;113:794-8.

4 Meyer UA. Personalized medicine: a personal view. Clin Pharmacol Ther. 2012;91:373-5.

5 Hoffrage U, et al. Medicine. Communicating statistical information. Science. 2000;290:2261-2.

6 Kurzenhauser S, Hertwig R. How to foster citizens' statistical reasoning: implications for genetic counseling. Community Genet. 2006;9:197-203.

7 Grassmann F, et al. Modelling the genetic risk in age-related macular degeneration. PLoS One. 2012;7:e37979.

8 Relkin NR, et al. The National Institute on Aging/ Alzheimer's Association recommendations on the application of apolipoprotein E genotyping to Alzheimer's disease. Ann N Y Acad Sci. 1996;802:149-76.

9 Seshadri S, et al. Genomewide analysis of genetic loci associated with Alzheimer disease. JAMA. 2010;303:1832-40.

10 Hirschhorn JN, Gajdos ZK. Genome-wide association studies: results from the first few years and potential implications for clinical medicine. Annu Rev Med. 2011;62:11-24.

11 Ripatti S, et al. A multilocus genetic risk score for coronary heart disease: case-control and prospective cohort analyses. Lancet. 2010;376: 1393-400.

12 Guttmacher AE, et al. The family history-more important than ever. N Engl J Med. 2004;351:2333-6.

13 Lee $\mathrm{W}$, et al. The mutation spectrum revealed by paired genome sequences from a lung cancer patient. Nature. 2010;465:473-7.

14 Ley TJ, et al. DNA sequencing of a cytogenetically normal acute myeloid leukaemia genome. Nature. 2008;456:66-72.

15 Pleasance ED, et al. A small-cell lung cancer genome with complex signatures of tobacco exposure. Nature. 2010;463:184-90.

16 Mateos-Caceres PJ, et al. Proteomic analysis of plasma from patients during an acute coronary syndrome. J Am Coll Cardiol. 2004;44:1578-83.

17 Sabatine MS, et al. Metabolomic identification of novel biomarkers of myocardial ischemia. Circulation. 2005; $112: 3868-75$.

18 Rosenberg S, et al. Multicenter validation of the diagnostic accuracy of a blood-based gene expression test for assessing obstructive coronary artery disease in nondiabetic patients. Ann Intern Med. 2010;153:425-34

19 Wingrove JA, et al. Correlation of peripheral-blood gene expression with the extent of coronary artery stenosis. Circ Cardiovasc Genet. 2008;1:31-8. 